\title{
C*-ALGEBRAS OF INVERSE SEMIGROUPS
}

\author{
by J. DUNCAN and A. L. T. PATERSON
}

(Received 10th January 1984)

\section{Introduction}

There are various algebras which may be associated with a discrete group $G$. In particular we may consider the complex group ring $\mathbb{C} G$, the convolution Banach algebra $l^{1}(G)$, the enveloping $C^{*}$-algebra $C^{*}(G)$ of $l^{1}(G)$, and the reduced $C^{*}$-algebra $C_{l}^{*}(G)$ determined by the completion of $l^{1}(G)$ under the left regular representation on $l^{2}(G)$. There is a substantial literature on the circle of ideas associated with the embeddings

$$
G \rightarrow \mathbb{C} G \rightarrow l^{1}(G) \rightarrow C^{*}(G) \rightarrow C_{l}^{*}(G) .
$$

There is also a growing literature on the corresponding problems when $G$ is replaced by a discrete semigroup $S$ with an involution. For an arbitrary involutive semigroup $S$ we may consider the embeddings

$$
S \rightarrow \mathbb{C} S \rightarrow l^{1}(S) \rightarrow C^{*}(S)
$$

but at this generality it is not clear how we should define the left regular representation on $l^{2}(S)$. There are good reasons for specialising to the case when $S$ is an inverse semigroup. The algebraic theory of inverse semigroups is well developed; moreover the class of such semigroups is precisely (up to isomorphism) the class of star subsemigroups of the set of partial isometries on a Hilbert space. As noted by Barnes [1] the Vagner-Preston representation of an inverse semigroup $S$ as partial one-one mappings on $S$ lifts to give a (star) representation $\lambda$ of $S$ and $l^{1}(S)$ on $l^{2}(S)$, and this is the appropriate definition for the left regular representation. Wordingham [10] has recently shown that $\lambda$ is always faithful on $l^{1}(S)$. Thus for any inverse semigroup $S$ we may consider the embeddings

$$
S \rightarrow \mathbb{C} S \rightarrow l^{1}(S) \rightarrow C^{*}(S) \rightarrow C_{l}^{*}(S),
$$

as the complete analogue of the group situation.

The mapping $G \mapsto C^{*}(G)$ does not classify groups up to isomorphism (not even for finite abelian groups); no more can the mapping $S \mapsto C^{*}(S)$ classify inverse semigroups up to isomorphism. Both mappings provide partial classifications as well as interesting examples of $C^{*}$-algebras. In his study of $l^{1}(S)$ for $S$ an inverse semigroup, Barnes [1], [2] provides some implicit information on $C^{*}(S)$ for some special classes of inverse 
semigroups. The second author [8] began an explicit study of $C^{*}(S)$ for the case in which $S$ is a Clifford semigroup. We here reconsider some of the problems discussed in [8] and we also initiate a much fuller programme of study of $C^{*}(S)$.

In Section 1 we present the key definitions and some technical results in the setting of an arbitrary inverse semigroup. We discuss the viewpoint on inverse semigroups as star semigroups of partial isometries on a Hilbert space, and note some of the important natural representations.

In Section 2 we specialise to the case in which $S$ is a Clifford $\omega$-semigroup $T$, i.e. a semilattice of groups in which the semilattice is $\left(\mathbb{Z}_{+}, \max \right)$. We identify $C^{*}(T)$ as a generalized sequence algebra associated with the $C^{*}$-algebras of the groups in $T$. For such Clifford semigroups we show that $T$ has weak containment (i.e. $C^{*}(T)=C_{l}^{*}(T)$ ) if and only if each group in $T$ is amenable.

In Section 3 we consider general inverse $\omega$-semigroups $\Omega$, i.e. inverse semigroups whose semilattice of idempotent elements is $\left(\mathbb{Z}_{+}, \max \right)$. We describe the (known) structure of such inverse semigroups, and we reduce the study of $C^{*}(\Omega)$ and the problem of weak containment for $\Omega$ to the case in which $\Omega$ is simple (or more specially, bisimple). This involves a detailed analysis of the Bruck-Reilly extension $\Omega$ of a finite union of groups $T$. For such $\Omega$ we describe $C^{*}(\Omega)$ in terms of a short exact sequence

$$
0 \rightarrow I \rightarrow C^{*}(\Omega) \rightarrow C^{*}\left(G_{\Omega}\right) \rightarrow 0
$$

where $I$ is the (unique) $C^{*}$-tensor product of $C^{*}(T)$ and the compact operators on a separable infinite dimensional Hilbert space, and where $G_{\Omega}$ is the maximal group homomorphic image of $\Omega$. We show that $\Omega$ has weak containment if and only if all its subgroups are amenable.

In the course of obtaining structure theorems for $C^{*}(S)$ we often obtain structure theorems for $\mathbb{C S}$ and most of the latter structure theorems may be generalised to $\mathbb{F} S$, for any field $\mathbb{F}$, with isomorphism in place of star isomorphism.

\section{Some general results}

A semigroup $S$ is an inverse semigroup if for each $s \in S$ there exists unique $s^{*} \in S$ with $s s^{*} s=s, s^{*} s s^{*}=s^{*}$. From now on, $S$ will always denote an inverse semigroup. A convenient introduction to inverse semigroups may be found in Howie [4], but we record here some facts that are fundamental to our study. The mapping $s \mapsto s^{*}$ is an involution on $S$, i.e. $s^{* *}=s$ and $(s t)^{*}=t^{*} s^{*}$ for all $s, t \in S$. We denote by $E_{S}$ the set of idempotents in $S$. Each idempotent of $S$ is self-adjoint, and $E_{S}$ is a commutative idempotent subsemigroup of $S$; in particular $E_{S}$ is a semilattice. An important congruence on $S$ is obtained by writing $s \sim t$ if $e s=e t$ for some $e \in E_{S}$. The quotient semigroup $S / \sim$ is then a group and it is the largest group homomorphic image of $S$. We write $G_{S}$ for $S / \sim$, and we denote the canonical homomorphism by $\chi: S \rightarrow G_{S}$. Evidently $\chi(e)=1$ for all $e \in E_{S}$, where 1 is the identity element of $G_{S} . S$ is said to be $E$-unitary if $\chi(s)=1$ implies $s \in E_{s}$. The structure of $E$-unitary inverse semigroups is well understood (see McAlister [5] for a survey) and they are more readily analysed than the others in the semigroup algebra setting (see e.g. [3]).

Inverse semigroups can always be viewed as semigroups of partial isometries on a 
Hilbert space. Recall that a bounded linear operator $U$ on a Hilbert space $\mathscr{H}$ is a partial isometry if $U U^{*} U=U$. We write $P I(\mathscr{H})$ for the set of all partial isometries on $\mathscr{H}$. In the Vagner-Preston representation an element of an inverse semigroup $S$ is represented as a partial one-one mapping on $S$ and hence may be represented as a partial isometry on the Hilbert space $l^{2}(S)$ as follows. For $s \in S$ let $\lambda(s)$ be the bounded linear operator on $l^{2}(S)$ determined by .

$$
\lambda(s) t= \begin{cases}s t & \text { if } s^{*} s t=t \\ 0 & \text { otherwise }\end{cases}
$$

Recall that there is a natural partial order on $S$ given by $s \leqq t$ if $s=e t$ for some $e \in E_{S}$ (equivalently $s=t f$ for some $f \in E_{S}$ ). When restricted to $E_{S}$ this gives the natural semilattice ordering on $E_{S}$, i.e. $e \leqq f$ if $e=e f$. Note that the condition $s^{*} s t=t$ is equivalent to the condition $t t^{*} \leqq s^{*} s$. A representation of $S$ on a Hilbert space $\mathscr{H}$ is a homomorphism $\pi: S \rightarrow \operatorname{PI}(\mathscr{H})$ with $\pi\left(s^{*}\right)=\pi(s)^{*}$ for all $s \in S$. (In fact if $\pi: S \rightarrow T$ is a homomorphism between inverse semigroups then the condition $\pi\left(s^{*}\right)=\pi(s)^{*}$ is automatic.)

\section{Proposition 1.1.}

(i) For any inverse semigroup $S, \lambda$ is a faithful representation of $S$ on $l^{2}(S)$, called the left regular representation.

(ii) If $\mathscr{S}$ is any star subsemigroup of $P I(\mathscr{H})$ then $\mathscr{S}$ is an inverse semigroup.

Proof. (i) is immediate from the Vagner-Preston theorem (see e.g. Howie [4, page 135]).

(ii) Several elementary proofs are available. For a brief proof note that a non-zero partial isometry has norm 1 and so an idempotent partial isometry is a projection. If $P, Q$ are projections with $P Q$ a partial isometry then $P Q$ is idempotent and so $P Q=Q P$. Thus $\mathscr{S}$ is a regular semigroup with commuting idempotents and so is an inverse semigroup (see e.g. Howie [4, page 130]).

For an arbitrary inverse semigroup there are two other natural representations which we note now. For a group $G$ let $\Lambda$ denote the left regular representation of $G$ on $l^{2}(G)$, i.e. $\Lambda(g) h=g h$. Then $\Lambda \circ \chi$ gives a representation of $S$ on $l^{2}\left(G_{S}\right)$. The universal representation of $S$ is obtained as the direct sum of "all" representations of $S$ (this is made more precise below). For certain representations of $S$ we can realise $G_{S}$ as follows.

Proposition 1.2. Let $\pi$ be a representation of $S$ on $\mathscr{H}$, and let $\mathscr{H}_{\infty}=\cap\left\{\pi(e) \mathscr{H}: e \in E_{S}\right\}$. Then $\pi(S) \mathscr{H}_{\infty} \subset \mathscr{H}_{\infty}$. Let $G_{\pi}=\left.\pi(S)\right|_{\mathscr{H}_{\infty}}$.

(i) $G_{\pi}$ is a group homomorphic image of $S$.

(ii) If $\pi$ contains $\Lambda \circ \chi$ as a direct summand, in particular if $\pi$ is the universal representation, then $G_{\pi}$ is (isomorphic to) $G_{\boldsymbol{S}}$.

Proof. Let $s \in S, \xi \in \mathscr{H}_{\infty}, e \in E_{S}$. Since $s^{*} e s \in E_{S}$ we have $\xi=\pi\left(s^{*} e s\right) \eta$ for $\eta \in \mathscr{H}$. Then $\pi(s) \xi=\pi(s) \pi\left(s^{*} e s\right) \eta=\pi\left(s s^{*} e s\right) \eta=\pi\left(e s s^{*} s\right) \eta=\pi(e) \pi(s) \eta$, so $\pi(s) \xi \in \mathscr{H}_{\infty}$. 
(i) Since $\left.\pi(s)\right|_{\mathscr{C}_{\infty}},\left.\pi\left(s^{*}\right)\right|_{\mathscr{H}_{\infty}}$ are isometries it follows that $\left.\pi(s)\right|_{\mathscr{C}_{\infty}}$ is unitary, as required.

(ii) Let $\pi$ contain $\Lambda \circ \chi$ as a direct summand. Evidently $l^{2}\left(G_{s}\right) \subset \mathscr{H}_{\infty}$ and so there is a homomorphism from $G_{\pi}$ onto $G_{S}$; but $G_{S}$ is the maximal group homomorphic image of $S$.

We now consider some of the algebras associated with $S$. $\mathbb{C S}$ is a (complex linear associative) star algebra and is a dense subalgebra of the Banach star algebra $l^{1}(S)$. $C^{*}(S)$ is the enveloping $C^{*}$-algebra of the Banach star algebra $l^{1}(S)$. Barnes [1] showed that $l^{1}(S)$ is an $A^{*}$-algebra so that the maximal $B^{*}$-seminorm $\|\circ\|$ on $l^{1}(S)$ is in fact a norm. For technical purposes later we take another viewpoint on $C^{*}(S)$ where we focus on $\mathbb{C S}$ rather than $l^{1}(S)$. There is some ambivalence in the literature as to the definition of a representation of an arbitrary star algebra $A$. One may define a representation to be a star homomorphism $\pi: A \rightarrow B L(\mathscr{H})$, where $B L(\mathscr{H})$ denotes the algebra of bounded linear operators on a Hilbert space. More generally one may define a representation of $A$ to be a star homomorphism $\pi: A \rightarrow L\left(\mathscr{H}_{0}\right)$, where $L\left(\mathscr{H}_{0}\right)$ denotes the algebra of linear operators on an inner product space $\mathscr{H}_{0}$. Palmer [7] calls a star algebra $A$ a $B G^{*}$ algebra when the above definitions are equivalent, i.e. when every star homomorphism $\pi: A \rightarrow L\left(\mathscr{H}_{0}\right)$ has the property that $\pi(A) \subset B L\left(\mathscr{H}_{0}\right)$. (Such algebras were introduced independently by Wordingham [11] under the name of uniform admissibility algebras, his definition being in terms of positive functionals.) Wordingham [11] notes that $\mathbb{C} S$ is a $B G^{*}$-algebra. (Let $\pi: \mathbb{C} S \rightarrow L\left(\mathscr{H}_{0}\right)$ be a star homomorphism. Then $\pi(e) \leqq I$ for $e \in E_{S}$. For $s \in S, \xi \in \mathscr{H}_{0}$ we have

$$
\|\pi(s) \xi\|^{2}=(\pi(s) \xi, \pi(s) \xi)=\left(\pi\left(s^{*} s\right) \xi, \xi\right) \leqq(\xi, \xi)
$$

so that $\pi(s) \in B L\left(\mathscr{H}_{0}\right)$ and so $\pi(\mathbb{C} S) \subset B L\left(\mathscr{H}_{0}\right)$.) The above argument also shows that $|\pi(a)| \leqq\|a\|_{1}$ for $a \in \mathbb{C} S$, where $\|\circ\|_{1}$ denotes the $l^{1}$-norm. In other words we may equally define $C^{*}(S)$ to be the completion of $\mathbb{C} S$ with respect to $\|\circ\|$, where

$$
\|a\|=\sup \left\{|\pi(a)|: \pi: \mathbb{C} S \rightarrow L\left(\mathscr{H}_{0}\right) \text { a star homomorphism }\right\} .
$$

This elementary observation would be of no great consequence were it not for the following useful facts about $B G^{*}$-algebras which we list without proof (see [7] and [11]).

Let $A$ be a $B G^{*}$-algebra. Then

(1) $\|a\|=\sup \left\{|\pi(a)|: \pi: A \rightarrow L\left(\mathscr{H}_{0}\right)\right.$ a star homomorphism $\}$ is finite for all $a \in A$ and gives the maximal $B^{*}$-seminorm on $A$.

(2) If $I$ is a $*$-ideal of $A$, then $I$ and $A / I$ are $B G^{*}$-algebras. Moreover every star representation of $I$ extends to a star representation of $A$.

(3) If $B$ is another $B G^{*}$-algebra then $A \otimes B$ is a $B G^{*}$-algebra.

To define $C^{*}(A)$ for an arbitrary $B G^{*}$-algebra one has to factor out the *-ideal $\{a:\|a\|=0\}$. In our applications this *-ideal is the zero ideal in all cases, so that $C^{*}(A)$ is just the completion of $A$ with respect to $\|\circ\|$. The following Proposition ([7], [11]) will be fundamental in later sections.

Proposition 1.3. Let $A$ be a $B G^{*}$-algebra and let I be a *-ideal of $A$. In the following 
diagram, in which all mappings are the natural ones, each square is commutative and the second row is exact.

$$
\begin{gathered}
0 \rightarrow \underset{\downarrow}{I} \rightarrow \underset{\downarrow}{A} \rightarrow A / I \rightarrow 0 \\
0 \rightarrow C^{*}(I) \rightarrow C^{*}(A) \rightarrow C^{*}(A / I) \rightarrow 0
\end{gathered}
$$

The algebra $C^{*}(S)$ is sometimes called the full $C^{*}$-algebra of $S$. The completion of $l^{1}(S)$ (or of $\mathbb{C} S$ ) with respect to the left regular representation norm, $|\lambda(\circ)|$, is called the reduced $C^{*}$-algebra of $S$ and is here denoted by $C_{l}^{*}(S)$. The left regular representation $\lambda$ extends to a star homomorphism $\lambda: C^{*}(S) \rightarrow C_{l}^{*}(S)$ and $\lambda$ is onto. Although $\lambda$ is faithful on $l^{1}(S)$ it need not be faithful on $C^{*}(S)$. We say that $S$ has weak containment if $\lambda$ is an isomorphism.

For some purposes it is convenient to take a spatial viewpoint on $C^{*}(S)$ and for this we use the universal representation. Since $l^{1}(S)$ need not have an identity nor even a bounded approximate identiry (see [3]), some care is needed in defining the universal representation. Since $\mathbb{C} S$ is a $B G^{*}$-algebra every positive functional $f$ on $\mathbb{C} S$ is admissible, i.e. gives rise to a star representation $\pi_{f}$ on a Hilbert space $\mathscr{H}_{f}$ (by the usual Gelfand-Naimark-Segal construction). Not every such $f$ gives rise to a cyclic (i.e. topologically cyclic) representation. The representation is cyclic if and only if $f$ is representable i.e. for some $\kappa>0|f(a)|^{2} \leqq \kappa f\left(a^{*} a\right)(a \in \mathbb{C} S)$. For representable $f$ there is a natural extension of $f$ to a positive functional on the unitization of $\mathbb{C S}$ obtained via

$$
f(1)=\inf \left\{\kappa:|f(a)|^{2} \leqq \kappa f\left(a^{*} a\right)(a \in \mathbb{C} S)\right\} .
$$

We say that $f$ is a state of $\mathbb{C} S$ if $f$ is a positive representable functional with $f(1)=1$. The universal representation of $\mathbb{C} S, \pi_{u}: \mathbb{C} S \rightarrow B L\left(\mathscr{H}_{u}\right)$ is the direct sum of all the star representations $\pi_{f}$ over all states of $\mathbb{C S}$, so that $\mathscr{H}_{u}=\Sigma^{\oplus} \mathscr{H}_{S}$. The universal representation of $S$ is the restriction of $\pi_{u}$ to $S$. We note that, for $a \in \mathbb{C} S$,

$$
\|a\|=\sup \left\{\left|\pi_{f}(a)\right|: f \text { a state of } \mathbb{C} S\right\} .
$$

(Every star representation of $\mathbb{C S}$ is a direct sum of an essential representation and a zero representation, and every essential representation is a direct sum of cyclic representations each of which is unitarily equivalent to $\pi_{f}$ for some state $f$.)

Any homomorphism $\sigma: S \rightarrow T$ is automatically a star homomorphism and extends to star homomorphisms $\sigma: \mathbb{C} S \rightarrow \mathbb{C} T, \sigma: l^{1}(S) \rightarrow l^{1}(T), \sigma: C^{*}(S) \rightarrow C^{*}(T)$. Unfortunately $\sigma$ need not extend to a star homomorphism $C_{l}^{*}(S) \rightarrow C_{l}^{*}(T)$, even in the case in which $S, T$ are groups. (For example let $S=F_{2}$, the free group on two symbols, $T=\mathbb{Z}$ and let $\sigma$ be the epimorphism obtained by mapping each of the symbols of $F_{2}$ to $1 \in \mathbb{Z}$ ). On the other hand the extension to $C_{l}^{*}(S)$ is always possible for the canonical homomorphism $\chi: S \rightarrow G_{S}$. This fact is implicit in the proof of Paterson [8, Proposition 4.1 (ii) $\Rightarrow$ (iii)] and we list it for later reference.

Proposition 1.4. The canonical homomorphism $\chi: S \rightarrow G_{S}$ extends to a star homomorphism $\chi: C_{l}^{*}(S) \rightarrow C_{l}^{*}\left(G_{s}\right)$. 


\section{Clifford $\omega$-semigroups}

A Clifford semigroup is an inverse semigroup $T$ which is a (disjoint) union of groups, or, equivalently, an inverse semigroup on which each idempotent is central. Any semilattice may act as the semilattice $E_{T}$ of a Clifford semigroup, but we shall here confine our attention to the case in which $E_{T}$ is (isomorphic to) $\omega$ where $\omega=\left(\mathbb{Z}_{+}, \vee\right)$ with

$$
m \vee n=\max \{m, n\} .
$$

A semigroup $S$ with $E_{S}=\omega$ is called an $\omega$-semigroup. Some, but by no means all, of the results which we present for Clifford $\omega$-semigroups have appropriate analogues for arbitrary Clifford semigroups. We confine our attention to $\omega$-semigroups for two reasons. The special case provides a useful introduction to the much more complicated general case, and it is precisely the case which we need for the next section when we consider arbitrary inverse $\omega$-semigroups. A discussion of $C^{*}$-algebras of arbitrary Clifford semigroups will appear elsewhere.

Throughout this section $T$ denotes a Clifford $\omega$-semigroup. Thus $T$ is a (disjoint) union of groups, $T=\cup\left\{G_{n}: n \in \mathbb{Z}_{+}\right\}$and $E_{T}=\left\{e_{n}: n \in \mathbb{Z}_{+}\right\}$where $e_{n}$ is the identity element of $G_{n}$. Note that the semilattice order on $E_{T}$ is the reverse of the usual order on $\mathbb{Z}_{+}$, i.e. $e_{m} \leqq e_{n}$ if and only if $m \geqq n$. Throughout the paper, for $m, n \in \mathbb{Z}_{+}, m \geqq n$ always denotes the usual order, i.e. $m-n \geqq 0$. The product in $T$ is described by a family of group homomorphisms. For $m \geqq n$ there is a homomorphism $Q_{m n}: G_{n} \rightarrow G_{m}$, and $\left\{Q_{m n}: m \geqq n\right\}$ is a coherent family, i.e.

(i) $Q_{n n}$ is the identity mapping on $G_{n}$,

(ii) $Q_{m n} Q_{n k}=Q_{m k}$ for $m \geqq n \geqq k$.

For $g_{m} \in G_{m}, g_{n}^{\prime} \in G_{n}$ we have

$$
g_{m} g_{n}^{\prime}=\left(Q_{j m} g_{m}\right)\left(Q_{j n} g_{n}^{\prime}\right)
$$

where $j=m \vee n$ and the product on the right takes place in $G_{j}$. The homomorphism $Q_{m n}$ has an internal description in $T$ by $Q_{m n} g_{n}=e_{m} g_{n}=g_{n} e_{m}$. We note that $e_{0}$ is the identity element of $T$.

Our first aim is to identify $C^{*}(T)$ as a sequence algebra determined by the family $\left\{C^{*}\left(G_{n}\right): n \in \mathbb{Z}_{+}\right\}$. We recall from Section 1 that each $Q_{m n}$ extends to a (norm-decreasing) star homomorphism $Q_{m n}: C^{*}\left(G_{n}\right) \rightarrow C^{*}\left(G_{m}\right)$.

Definition 2.1. For $n \in \mathbb{Z}_{+}$let $A_{n}$ be a $C^{*}$-algebra with norm $|\circ|_{n}$. Let $\left\{Q_{m n}: A_{n} \rightarrow A_{m}: m \geqq n\right\}$ be a coherent family of star homomorphisms. We denote by $\mathscr{S}\left\{A_{n}\right\}$ the $C^{*}$-algebra of all bounded sequences $\left\{x_{n}\right\}$, where $x_{n} \in A_{n}\left(n \in \mathbb{Z}_{+}\right)$, with pointwise operations and supremum norm

$$
\left\|\left\{x_{n}\right\}\right\|_{\infty}=\sup _{n}\left|x_{n}\right|_{n} .
$$

We denote by $\mathscr{C}\left\{A_{n}\right\}$ the $C^{*}$-subalgebra of $\mathscr{P}\left\{A_{n}\right\}$ consisting of all sequences $\left\{x_{n}\right\}$ such 
that

$$
\lim _{n \rightarrow \infty} \sup _{m \geqq n}\left|x_{m}-Q_{m n} x_{n}\right|_{m}=0 .
$$

For each $j \in \mathbb{Z}_{+}$there is a natural (isometric) star monomorphism of $A_{j}$ into $\mathscr{C}\left\{A_{n}\right\}$ defined by $a \mapsto\left\{x_{n}\right\}$ where

$$
x_{n}=\left\{\begin{array}{cl}
Q_{n j} a & \text { if } n \geqq j \\
0 & \text { otherwise }
\end{array}\right.
$$

For $T=\cup\left\{G_{n}: n \in \mathbb{Z}_{+}\right\}$we apply the above construction with $A_{n}=C^{*}\left(G_{n}\right)$, the norm in $C^{*}\left(G_{n}\right)$ being denoted by $\|\circ\|_{n}$. We regard $\mathbb{C} G_{m}$ as embedded in $C^{*}\left(G_{m}\right)$ and hence in $\mathscr{C}\left\{C^{*}\left(G_{n}\right)\right\}$ via the above monomorphism. Let $\tau: \mathbb{C} T \rightarrow \mathscr{C}\left\{C^{*}\left(G_{n}\right)\right\}$ be the linear extension of all the embeddings $\mathbb{C} G_{m} \rightarrow \mathscr{C}\left\{C^{*}\left(G_{n}\right)\right\}$. We denote the norm in $C^{*}(T)$ by $\|\circ\|$ and we regard $\mathbb{C} T$ as embedded in $C^{*}(T)$. For $a \in \mathbb{C} G_{m}$ we must distinguish, a priori, $\|a\|_{m}$ and $\|a\|$; fortunately these norms coincide.

Lemma 2.2. For $a \in \mathbb{C} G_{m}$ we have $\|a\|=\|a\|_{m}$.

Proof. The natural injection $\alpha: G_{m} \rightarrow T$ extends to a star homomorphism $\alpha: C^{*}\left(G_{m}\right) \rightarrow C^{*}(T)$. Let $\beta: \mathbb{C} T \rightarrow \mathbb{C} G_{m}$ be the linear mapping determined by

$$
\beta\left(g_{n}\right)=\left\{\begin{array}{cl}
Q_{m n} g_{n} & \text { if } m \geqq n \\
0 & \text { otherwise }
\end{array}\right.
$$

For $m \geqq i \vee j$ we have

$$
Q_{m i \vee j} g_{i} g_{j}=\left(Q_{m i} g_{i}\right)\left(Q_{m j} g_{j}\right)
$$

and hence $\beta\left(g_{i} g_{j}\right)=\beta\left(g_{i}\right) \beta\left(g_{j}\right)$. It follows that $\beta$ is a star homomorphism and so extends to a star homomorphism $\beta: C^{*}(T) \rightarrow C^{*}\left(G_{m}\right)$. But $\beta\left(\alpha\left(g_{m}\right)\right)=g_{m}$, so that $\alpha$ is one-one and therefore isometric, as required.

Lemma $2.3 \tau: \mathbb{C} T \rightarrow \mathscr{C}\left\{C^{*}\left(G_{n}\right)\right\}$ is a star homomorphism with dense range.

Proof. Clearly $\tau\left(a^{*}\right)=\tau(a)^{*}$ for $a \in \mathbb{C} T$. To show that $\tau$ is a homomorphism it is enough to show that

$$
\tau\left(g_{j} g_{k}\right)=\tau\left(g_{j}\right) \tau\left(g_{k}\right)
$$

For $n \geqq j \vee k$ we have

$$
\begin{aligned}
\tau\left(g_{j} g_{k}\right)_{n} & =Q_{n j \vee k} g_{j} g_{k} \\
& =\left(Q_{n j} g_{j}\right)\left(Q_{n k} g_{k}\right) \\
& =\tau\left(g_{j}\right)_{n} \tau\left(g_{k}\right)_{n}
\end{aligned}
$$


and otherwise

$$
\tau\left(g_{j} g_{k}\right)_{n}=0=\tau\left(g_{j}\right)_{n} \tau\left(g_{k}\right)_{n}
$$

Therefore $\tau$ is a star homomorphism, and so is continuous.

Let $\left\{x_{n}\right\} \in \mathscr{C}\left\{C^{*}\left(G_{n}\right)\right\}$. Then

$$
\left\{x_{n}\right\}=\tau\left(x_{0}\right)+\sum_{j=1}^{k} \tau\left(x_{j}-Q_{j j-1} x_{j-1}\right)+\left\{y_{n}\right\}
$$

where

$$
y_{n}=\left\{\begin{array}{ll}
0 & n \leqq k \\
x_{n}-Q_{n k} x_{k} & n>k
\end{array} .\right.
$$

By the definition of $\mathscr{C}\left\{C^{*}\left(G_{n}\right)\right\}$ we have $\left\|\left\{y_{n}\right\}\right\|_{\infty} \rightarrow 0$ as $k \rightarrow \infty$. Since $\mathbb{C} G_{j}$ is dense in $C^{*}\left(G_{j}\right)$ it follows that $\tau(\mathbb{C} T)$ is dense in $\mathscr{C}\left\{C^{*}\left(G_{n}\right)\right\}$.

It is immediate from Lemma 2.3 that $\tau$ extends to a star epimorphism $\tau: C^{*}(T) \rightarrow \mathscr{C}\left\{C^{*}\left(G_{n}\right)\right\}$. We show now that $\tau$ is an isomorphism; of the two proofs known to us both involve some degree of computation.

Theorem 2.4. Let $T$ be the Clifford $\omega$-semigroup $\left\{G_{n}: n \in \mathbb{Z}_{+}\right\}$. Then $\tau: C^{*}(T) \rightarrow \mathscr{C}\left\{C^{*}\left(G_{n}\right)\right\}$ is a star isomorphism.

Proof. In view of Lemma 2.3 it is enough to prove that $\|h\| \leqq\|\tau(h)\|_{\infty}$ for $h \in \mathbb{C} T$, $h^{*}=h$, or equivalently that $|\sigma(h)| \leqq\|\tau(h)\|_{\infty}$ for any star representation $\sigma$ of $\mathbb{C} T$. Let $h=\sum_{j=0}^{k} h_{j}$ where $h_{j} \in \mathbb{C} G_{j}, h_{j}^{*}=h_{j}$. Since $\left.\sigma\right|_{c G_{j}}$ is a star representation we note that $\left|\sigma\left(a_{j}\right)\right| \leqq\left\|a_{j}\right\|_{j}$ for $a_{j} \in \mathbb{C} G_{j}$.

We show that $\lim _{n \rightarrow \infty}\left|\sigma\left(h^{n}\right)\right|^{1 / n} \leqq\|\tau(h)\|_{\infty}$. In the expansion of $h^{n}$ the terms are partitioned amongst $\mathbb{C} G_{0}, \mathbb{C} G_{1}, \ldots, \mathbb{C} G_{k}$. By adding and subtracting terms we obtain the following "homogeneous" expression for $h^{n}$, viz

$$
h^{n}=h_{0}^{n}+\sum_{j=1}^{k}\left\{\left(Q_{j 0} h_{0}+Q_{j 1} h_{1}+\cdots+h_{j}\right)^{n}-\left(Q_{j 0} h_{0}+Q_{j 1} h_{1}+\cdots+Q_{j j-1} h_{j-1}\right)^{n}\right\}
$$

whence

$$
\left|\sigma\left(h^{n}\right)\right| \leqq\left\|h_{0}\right\|_{0}^{n}+\sum_{j=1}^{k}\left\{\left\|Q_{j 0} h_{0}+\cdots+h_{j}\right\|_{j}^{n}+\left\|Q_{j 0} h_{0}+\cdots+Q_{j j-1} h_{j-1}\right\|^{n}\right\} .
$$

Therefore

$$
\begin{aligned}
|\sigma(h)| & =\lim _{n \rightarrow \infty}\left|\sigma\left(h^{n}\right)\right|^{1 / n} \\
& \leqq \max \left\{\left\|h_{0}\right\|_{0},\left\|Q_{10} h_{0}+h_{1}\right\|_{1},\left\|Q_{10} h_{0}\right\|_{1}, \ldots,\right.
\end{aligned}
$$




$$
\begin{aligned}
& \left.\left\|Q_{k 0} h_{0}+\cdots+h_{k}\right\|_{k},\left\|Q_{k 0} h_{0}+\cdots+Q_{k k-1} h_{k-1}\right\|_{k}\right\} \\
= & \|\tau(h)\|_{\infty} .
\end{aligned}
$$

This completes the proof.

Remark. Suppose that $T$ is commutative, so that each group $G_{n}$ is abelian. Let $T^{\wedge}$ denote the set of semicharacters of $T$. Then $C^{*}(T)$ is star isomorphic to $C\left(T^{\wedge}\right), T^{\wedge}$ being compact under the topology of pointwise convergence. The space $T^{\wedge}$ is rather intricate, but Theorem 2.4 gives a star isomorphism $\tau: C^{*}(T) \rightarrow \mathscr{C}\left\{C^{*}\left(G_{n}\right)\right\}=\mathscr{C}\left\{C\left(G_{n}^{\wedge}\right)\right\}$ in terms of sequences of continuous functions on simpler spaces.

We record here for later use some easy variants of Theorem 2.4. With the obvious modification Theorem 2.4 holds for Clifford semigroups $T$ for which $E_{T}$ is a finite subsemilattice of $\omega$ (i.e. $E_{T}$ is a finite chain); for this case $\mathscr{C}\left\{C^{*}\left(G_{n}\right)\right\}$ consists of all finite sequences. The concept of Clifford semigroup, i.e. semilattice of groups, may be generalised to that of a semilattice of inverse semigroups. We restrict attention to the case in which the semilattice is $\omega$. Even for the $\omega$-case there are two possible concepts. In the usual definition (see [4], page 89) one takes $S=\cup\left\{S_{n}: n \in \mathbb{Z}_{+}\right\}$, the union being disjoint, where the $S_{n}$ are inverse semigroups and $S_{m} S_{n} \subset S_{m \vee n}$. For our purposes we use the more restricted concept that $S=\cup\left\{S_{n}: n \in \mathbb{Z}_{+}\right\}$with multiplication determined by a coherent family of homomorphisms $\left\{Q_{m n}: S_{n} \rightarrow S_{m}: m \geqq n\right\}$. The two concepts coincide when each $S_{n}$ has identity element, say $u_{n}$, and $\left\{u_{n}: n \in \mathbb{Z}_{+}\right\}$is a subsemigroup; for then we may take $Q_{m n} s_{n}=u_{m} s_{n}$, noting that each $u_{m}$ is central since

$$
u_{m} s_{n}=u_{m}\left(u_{m} s_{n}\right)=\left(u_{m} s_{n}\right) u_{m}=u_{m}\left(s_{n} u_{m}\right)=\left(s_{n} u_{m}\right) u_{m}=s_{n} u_{m},
$$

and also that $Q_{p m} Q_{m n}=Q_{p n}$ for $p \geqq m \geqq n$ since $u_{p} u_{m} u_{n}=u_{p} u_{n}=u_{n}$ for $p \geqq m \geqq n$. Theorem 2.4 generalises as listed below, the construction and proof proceeding exactly as before with $G_{n}$ replaced by $S_{n}$. We remark that Theorem 2.5 holds when $\omega$ is replaced by a finite chain, and this fact will be used in Section 3.

Theorem 2.5 Let $S_{n}$ be an inverse semigroup for each $n \in \mathbb{Z}_{+}$and let $S=\cup\left\{S_{n}: n \in \mathbb{Z}_{+}\right\}$ be the inverse semigroup determined by a coherent family of homomorphisms $\left\{Q_{m n}: m \geqq n\right\}$. Then $\tau: C^{*}(S) \rightarrow \mathscr{C}\left\{C^{*}\left(S_{n}\right)\right\}$ is a star isomorphism.

We turn now to the weak containment problem for $T$.

Theorem 2.6. Let $T$ be the Clifford $\omega$-semigroup $\cup\left\{G_{n}: n \in \mathbb{Z}_{+}\right\}$. Then $T$ has weak containment is and only if each $G_{n}$ is amenable.

Proof. This follows directly from [8, Proposition 3.7(ii)] or by a straightforward application of Theorem 2.4 .

Similarly we obtain the following result. 
Theorem 2.7. Let $S$ be as in Theorem 2.5. Then $S$ has weak containment if and only if each $S_{n}$ has weak containment.

We remark that Theorems $2.6,2.7$ hold when $\omega$ is replaced by a finite chain.

\section{Inverse $\omega$-semigroups}

Throughout this section $\Omega$ will denote an inverse $\omega$-semigroup, i.e. an inverse semigroup $\Omega$ with $E_{\Omega}=\omega$. As in Section 2 we write $E_{\Omega}=\left\{e_{n}: n=0,1,2, \ldots\right\}$ and we have

$$
e_{0}>e_{1}>e_{2}>\cdots
$$

and also that $e_{0}$ is the identity element of $\Omega$. The most important example of an inverse $\omega$-semigroup which is not a Clifford $\omega$-semigroup is provided by the bicyclic semigroup which we here denote by $\mathscr{C}$. Recall that $\mathscr{C}$ may be regarded as the semigroup generated by $p, q$ subject to the relation $q p=1$. Thus

$$
\mathscr{C}=\left\{p^{m} q^{n}: m, n=0,1,2, \ldots\right\} \quad \text { and } \quad E_{\mathscr{C}}=\left\{p^{n} q^{n}: n=0,1,2, \ldots\right\}
$$

We note that $\left(p^{m} q^{n}\right)^{*}=p^{n} q^{m}$. When it is convenient we denote $p^{m} q^{n}$ as $(m, n)$; the multiplication in $\mathscr{C}$ is then given by

$$
(m, n)(i, j)=(m-n+\max (n, i), j-i+\max (n, i)) .
$$

There is a complete classification of inverse $\omega$-semigroups in terms of appropriate building blocks (see Munn [6]). We approach the classification from a slightly different viewpoint from Munn, and so we give a brief sketch of the preliminary analysis although the arguments are implicit in [6]. Even this brief sketch shows how critical to the argument is the structure of the semilattice $\omega$. The key definition for the classification is the following.

Definition 3.1. Idempotents $e, f$ of an inverse semigroup $S$ are $\mathscr{D}$-equivalent, written $e \mathscr{D} f$, if there exists $x \in S$ such that $x x^{*}=e, x^{*} x=f$.

Let $\Omega$ be an inverse $\omega$-semigroup such that $e_{0} \mathscr{D} e_{1}$. Let $e_{0}=v u, e_{1}=u v$ with $v=u^{*}$. Clearly $u v>u^{2} v^{2}$. If $u v>e_{2}>u^{2} v^{2}$ then $v u v u>v e_{2} u>v u^{2} v^{2} u$, i.e. $e_{0}>v e_{2} u>e_{1}$, which is impossible. Thus $u^{2} v^{2}=e_{2}$, and more generally $u^{n} v^{n}=e_{n}$. Since $v^{n} u^{n}=e_{0}$, it follows that all idempotents of $\Omega$ are $\mathscr{D}$-equivalent, so that $\Omega$ is a bisimple inverse $\omega$-semigroup.

Let $\Omega$ be an inverse $\omega$-semigroup such that $e_{0} \mathscr{D} e_{d}$ where $d(\geqq 2)$ is minimal. Let $e_{0}=v u, e_{d}=u v$ with $v=u^{*}$. An elaboration of the above argument shows that $u e_{k} v=e_{d+k}$ for $k=1,2, \ldots, d$, and thence that $e_{k} \mathscr{D} e_{n d+k}$ for $n=1,2,3, \ldots$ Thus $\Omega$ is a simple inverse $\omega$-semigroup.

Suppose now that $\Omega$ is an inverse $\omega$-semigroup such that $e_{0} \mathscr{D} e_{d}$ is false for all $d \neq 0$. There are two cases. If no two idempotents of $\Omega$ are $\mathscr{D}$-equivalent then $x^{*} x=x x^{*}$ for all $x \in \Omega$, so that $\Omega$ is a union of groups and so $\Omega$ is a Clifford $\omega$-semigroup. Alternatively 
we may choose $k$ and $d$ to be minimal with $e_{k} \mathscr{D} e_{k+d}$. Then $\Omega$ is a finite chain of inverse semigroups of the form $G_{0} \cup G_{1} \cup \cdots \cup G_{k-1} \cup \Omega_{k}$ where each $G_{i}$ is a group and $\Omega_{k}$ is a simple (bisimple if $d=1$ ) inverse $\omega$-semigroup. The first two cases above may be included in this latter case if we adopt the convention that $k=0$ denotes that no groups are present.

The above provides a complete classification of inverse $\omega$-semigroups (modulo the structure of the bisimple and simple cases which we discuss below). Recall that the analogues of Theorems 2.5 and 2.7 hold for finite subsemilattices of $\omega$, and note that, for $0 \leqq j \leqq k-1, e_{j} e_{k}=e_{k}$ where $e_{k}$ is the identity element of $\Omega_{k}$ (else $\Omega$ would not be an $\omega$-semigroup). Thus we have the following theorem.

Theorem 3.2. Let $\Omega$ be an inverse $\omega$-semigroup which is not a Clifford $\omega$-semigroup, so that $\Omega$ is a finite chain of the form $G_{0} \cup G_{1} \cup \cdots \cup G_{k-1} \cup \Omega_{k}$ where $\Omega_{k}$ is simple (or bisimple).

(i) $C^{*}(\Omega)$ is star isomorphic to the finite sequence algebra determined by $C^{*}\left(G_{i}\right)$ $i=0,1, \ldots, k-1$ and $C^{*}\left(\Omega_{k}\right)$.

(ii) $\Omega$ has weak containment if and only if $G_{i}$ is amenable for $i=0,1, \ldots, k-1$ and $\Omega_{k}$ has weak containment.

It remains to analyse in detail the bisimple and simple cases. We begin by describing the structure of the semigroups and then consider their $C^{*}$-algebras.

Definition 3.3. Let $T$ be an inverse semigroup with identity element and let $\alpha$ be a homomorphism from $T$ to its group of units (i.e. the maximal subgroup of $T$ whose identity is the identity element of $T$ ). Let $B R(T, \alpha)=T \times \mathscr{C}$ with multiplication defined by

$$
(s, m, n)(t, i, j)=\left(\left(\alpha^{k-n} s\right)\left(\alpha^{k-i} t\right), m-n+k, j-i+k\right)
$$

where $k=\max (n, i)$ and where $\alpha^{0}$ is the identity mapping on $T . B R(T, \alpha)$ is called the Bruck-Reilly extension of $T$ determined by $\alpha$.

For any inverse semigroup $T$ and any $\alpha, B R(T, \alpha)$ is a simple inverse semigroup. Now let $T$ be a group $G$ and let $\alpha$ be an endomorphism of $G$. Then $B R(G, \alpha)$ is a bisimple inverse $\omega$-semigroup; conversely any bisimple inverse $\omega$-semigroup is isomorphic to some $B R(G, \alpha)$. Now let $T$ be a Clifford semigroup whose semilattice is a finite chain. Then $B R(T, \alpha)$ is a simple inverse $\omega$-semigroup; conversely any simple inverse $\omega$ semigroup is isomorphic to some $B R(T, \alpha)$ for such a $T$. These results are all conveniently expounded in Howie [4, Chapter V] who also notes the following alternative formulation of the simple case. Let $T=G_{0} \cup G_{1} \cup \cdots \cup G_{d-1}$ and let the coherent family of group homomorphisms be determined by compositions of the homomorphisms

$$
\gamma_{i}: G_{i} \rightarrow G_{i+1} \quad(i=0,1, \ldots, d-2) .
$$

Let $\gamma_{d-1}: G_{d-1} \rightarrow G_{0}$ be a homomorphism. Then in the representation $B R(T, \alpha)$ for a 
simple inverse $\omega$-semigroup the homomorphism $\alpha: T \rightarrow G_{0}$ takes the form

$$
\left.\alpha\right|_{G_{i}}=\gamma_{i} \gamma_{i+1} \ldots \gamma_{d-1} \quad(i=0,1, \ldots, d-1) .
$$

It is central to our analysis of $C^{*}(\Omega)$ to have a detailed description of the canonical homomorphism $\chi: \Omega \rightarrow G_{\Omega}$. The congruence $\sim$ is described in the following elementary lemma whose proof we omit. It is well known that $G_{\mathscr{G}}=\mathbb{Z}$ with $\chi(m, n)=m-n$.

Lemma 3.4. Let $S=B R(T, \alpha)$ for some inverse semigroup $T$.

(i) $E_{S}=E_{T} \times E_{\mathscr{G}}$.

(ii) $(t, m, n) \sim(u, i, j)$ in $S$ if and only if $(m, n) \sim(i, j)$ in $\mathscr{C}$ (i.e. $m-n=i-j)$ and $f \alpha^{k-m} t=$ $f \alpha^{k-i} u$ for some $f \in E_{T}$ and some integer $k \geqq \max (m, i)$.

Remark 1. Let $\Omega=B R(T, \alpha)$ with $T=\bigcup_{i=0}^{d-1} G_{i}$ and associated homomorphisms $\gamma_{i}$ $(i=0,1, \ldots, d-1)$. It follows easily from Lemma 3.4 that $\Omega$ is $E$-unitary if and only if each $\gamma_{i}$ is one-one. In particular, $B R(G, \alpha)$ is $E$-unitary if and only if $\alpha$ is one-one.

Remark 2. We take this opportunity to present some folklore concerning $B R(G, \alpha)$ when $\alpha \in$ Aut $G$, i.e. $\alpha$ is an automorphism of $G$. For this case, $B R(G, \alpha)$ may be described in the more familiar language of crossed products. Let $G, H$ be groups and let $\beta: H \rightarrow$ Aut $G$ be a homomorphism. The crossed product $G \times{ }_{\beta} H$ is the group $\{g \otimes h: g \in G, h \in H\}$ with multiplication

$$
\left(g_{1} \otimes h_{1}\right)\left(g_{2} \otimes h_{2}\right)=g_{1} \beta\left(h_{1}\right)\left(g_{2}\right) \otimes h_{1} h_{2}
$$

Now let $K$ be an inverse semigroup and let $\beta: K \rightarrow$ Aut $G$ be a homomorphism. Since Aut $G$ is a group, $\beta$ must factor through $G_{K}$, so that $\beta$ induces a homomorphism $\tilde{\beta}: G_{K} \rightarrow$ Aut $G$ by $\widetilde{\beta}(\chi(k))=\beta(k)$. We may define the crossed product $G \times{ }_{\beta} K$ analogously to the group case. Then $S=G \times{ }_{\beta} K$ becomes an inverse semigroup with

$$
(g \otimes k)^{*}=\left(\beta\left(k^{*}\right)\left(g^{-1}\right) \otimes k^{*}\right)
$$

It is elementary to verify that $E_{S}=\left\{1 \otimes e: e \in E_{K}\right\}$, that $G_{S}$ is isomorphic to $G \times{ }_{\beta} G_{K}$, and that $S$ is $E$-unitary if and only if $K$ is $E$-unitary. We now record the link between $B R(G, \alpha)$ and crossed products.

Proposition 3.5. Let $\Omega=B R(G, \alpha)$ with $\alpha \in$ Aut $G$ and let $\beta: \mathscr{C} \rightarrow$ Aut $G$ be given by $\beta(c)=\alpha^{-\chi(c)}$.

(i) $B R(G, \alpha)$ is isomorphic to $G \times{ }_{\beta} \mathscr{C}$.

(ii) $G_{\Omega}$ is isomorphic to $G \times \mathbb{p} \mathbb{Z}$, and so is amenable if and only if $G$ is amenable.

Proof. (i) The mapping $G \times{ }_{\beta} \mathscr{C} \rightarrow B R(G, \alpha)$ given by

$$
g \otimes c \mapsto\left(g, e_{0}\right)(1 \otimes c)
$$

provides the required isomorphism. 
(ii) This follows from (i), the above remarks, and the well known properties of amenable groups.

Part (i) of Proposition 3.5 does not seem to generalise but there is a complete generalisation of part (ii) that describes $G_{\Omega}$ for an arbitrary simple inverse $\omega$-semigroup $\Omega$. The proof makes key use of a construction whereby a monomorphism $\alpha: G \rightarrow G$ is extended to an automorphism $\bar{\alpha}$ of a group $G^{\alpha}$ which is a countable union of subgroups each isomorphic to $G$.

Proposition 3.6. Let $\Omega=B R(T, \alpha)$ with $T=\bigcup_{i=0}^{d-1} G_{i}$ and associated homomorphisms $\gamma_{i}$ $(i=0,1, \ldots, d-1)$.

(i) There is a group $W$ and a homomorphism $\beta: \mathbb{Z} \rightarrow$ Aut $W$ such that $G_{\Omega}$ is isomorphic to $W \times{ }_{\beta} \mathbb{Z}$.

(ii) $G_{\Omega}$ is amenable if some $G_{i}$ is amenable (equivalently all $G_{i}$ are amenable).

(iii) If $\Omega$ is E-unitary, then all $G_{i}$ are amenable if $G_{\Omega}$ is amenable.

Proof. We write $z_{i}$ for the identity element of the group $G_{i}$, so that $z_{0}$ is the identity element of the semigroup $T$. We write $[t, m, n]$ for the $\sim$-equivalence class of $(t, m, n)$ in $\Omega$. Let $\rho[t, m, n]=m-n$, so that $\rho: G_{\Omega} \rightarrow \mathbb{Z}$ is a homomorphism. Let $W=\operatorname{ker} \rho$, so that $W$ is a normal subgroup of $G_{\mathbf{\Omega}}$ and

$$
W=\left\{[t, k, k]: t \in T, k \in \mathbb{Z}_{+}\right\} .
$$

Let $Z=\left\{\left[z_{0}, m, n\right]:(m, n) \in \mathscr{C}\right\}$. Then $Z$ is a subgroup of $G_{\Omega}$ and $Z$ is isomorphic to $\mathbb{Z}$. Note in fact (from Lemma 3.4) that $\left[z_{0}, m, n\right]=\left[z_{0}, i, j\right]$ if and only if $(m, n) \sim(i, j)$ in $\mathscr{C}$, so that $\left[z_{0}, r, r\right]$ is the identity element of $G_{\Omega}$ (for any $r \in \mathbb{Z}_{+}$). It follows (again by Lemma 3.4) that $W \cap Z$ is the trivial subgroup of $G_{\Omega}$. Since $(t, m, n)=(t, m, m)\left(z_{0}, m, n\right)$ we have

$$
[t, m, n]=[t, m, m]\left[z_{0}, m, n\right]
$$

and so $G_{\Omega}=W Z$. We have now established that $G_{\Omega}$ is a split extension of $W$ by $Z$. This proves (i). In fact we readily identify the mapping that implements the crossed product. By the well known link between split extensions and crossed products we have

$$
\beta(\zeta) w=\zeta w \zeta^{-1}(\zeta \in Z, w \in W)
$$

Identify $Z$ with $\mathbb{Z}$ and we find by a routine computation that $\beta(n)=\tilde{\alpha}^{-n}(n \in \mathbb{Z})$ where

$$
\tilde{\alpha}[t, k, k]=[\alpha t, k, k] \text {. }
$$

It is now clear that $G_{\Omega}$ is amenable if and only if $W$ is amenable. To establish (ii) and (iii) we need to see how $W$ is determined by $T$ and $\alpha$. It is convenient to begin with the case $T=G$. [In this case $W=\lim _{n} G_{n}$, where $G_{n}=G$ for all $n$ and each homomorphism $G_{n} \rightarrow G_{n+1}$ is given by $\left.\alpha\right]$.

Let $T=G$ and suppose $\alpha$ is onto. Then $W=\{[t, 0,0]: t \in G\}$. Since $(t, 0,0) \sim\left(t^{\prime}, 0,0\right)$ if 
and only if $\alpha^{r} t=\alpha^{r} t^{\prime}$ for some $r \in \mathbb{Z}_{+}$it follows that $W$ is isomorphic to $G / N^{\alpha}$ where $N^{\alpha}=\cup\left\{\operatorname{ker} \alpha^{n}: n \in \mathbb{Z}_{+}\right\}$. (If $\alpha$ is one-one then $W$ is isomorphic to $G$, as in Proposition 3.5). In fact the $W$ for $B R(G, \alpha)$ is isomorphic to the corresponding $W$ for $B R\left(G / N^{\alpha}, \hat{\alpha}\right)$ where $\hat{\alpha}([g])=[\alpha g]$, so that $\hat{\alpha}$ is one-one. Thus, for $T=G$ it is now enough to analyse the case in which $\alpha$ is one-one. For this case we let

$$
W_{n}=\{[t, k, k]: t \in G, k=0,1, \ldots, n\} .
$$

Then $W_{n}$ is a subgroup of $W$. Define $\sigma_{n}: G \rightarrow W_{n}$ by $\sigma_{n}(t)=[t, n, n]$. Then $\sigma_{n}$ is a homomorphism, $\sigma_{n}$ is one-one since $\alpha$ is one-one, and $\sigma_{n}$ is onto since $[t, k, k]=\left[\alpha^{n-k} t, n, n\right]$. Thus $W$ is the increasing union of the groups $W_{n}$, each of which is isomorphic to $G$.

Now let $T=\bigcup_{i=0}^{d-1} G_{i}$. For each $i$ we obtain an endomorphism $\alpha_{i}: G_{i} \rightarrow G_{i}$ by following round all the $\gamma_{i}$, i.e.

$$
\alpha_{i}=\gamma_{i-1} \ldots \gamma_{0} \gamma_{d-1} \ldots \gamma_{i+1} \gamma_{i} .
$$

With $t, t^{\prime} \in G_{i}$, we readily verify that $(t, k, k) \sim\left(t^{\prime}, k, k\right)$ if and only if $\alpha_{i}^{r} t=\alpha_{i}^{r} t^{\prime}$ for some $r \in \mathbb{Z}_{+}$. As above we write $N^{\alpha_{i}}=\cup\left\{\operatorname{ker} \alpha_{i}^{n}: n \in \mathbb{Z}_{+}\right\}$. Since $\gamma_{i} N^{\alpha_{i}} \subset N^{\alpha_{i+1}}$ we get an induced monomorphism $\hat{\gamma}_{i}: G_{i} / N^{\alpha_{i}} \rightarrow G_{i+1} / N^{\alpha_{i+1}}$. To determine $W$ we may thus replace $G_{i}$ and $\gamma_{i}$ by $G_{i} / N^{\alpha_{i}}$ and $\hat{\gamma}_{i}$. In other words we are reduced to analysing the case in which each $\gamma_{i}$ is one-one. For this case note that $\left(g_{i}, k, k\right) \sim\left(g_{i+r}, k, k\right)$ means that $g_{i+r}=z_{r} g_{i}$. Let

$$
W_{n}=\{[t, k, k]: t \in T, \quad k=0,1, \ldots, n\}
$$

so that $W=\cup\left\{W_{n}: n \in \mathbb{Z}_{+}\right\}$. By the above fact

$$
W_{n}=\left\{[t, k, k]: t \in G_{d-1}, \quad k=0,1, \ldots, n\right\}
$$

and $W_{n}$ is isomorphic to $G_{d-1}$, as in the group case above.

Parts (ii) and (iii) follow from the above analysis of $W$. The fact that each $G_{i}$ is amenable if and only if some $G_{i}$ is amenable follows from the fact that each $G_{i}$ may be mapped homomorphically into any other $G_{i}$. Evidently, the condition that $\Omega$ be $E$ unitary cannot be omitted in (iii).

We turn now to the description of $C^{*}(\Omega)$ for the simple (and bisimple) case. For the case $\Omega=\mathscr{C}$, the canonical homomorphism $\chi: \mathscr{C} \rightarrow \mathbb{Z}$ extends to $\chi: C^{*}(\mathscr{C}) \rightarrow C^{*}(\mathbb{Z})$. (It is well known that $C^{*}(\mathbb{Z})=C(\mathbb{T})$ where $\mathbb{T}$ is the circle group). The kernel of $\chi$ in $C^{*}(\mathscr{C})$ is star isomorphic to $\mathscr{K}$, the $C^{*}$-algebra of compact operators on the Hilbert space $l^{2}$. This last fact has several proofs but it will follow in particular as a special case of Theorem 3.9 below. For the general case $\Omega=B R(T, \alpha)$ we let $J$ be the kernel of $\chi: \mathbb{C} \Omega \rightarrow \mathbb{C} G_{\Omega}$. Proposition 1.3 then gives us a short exact sequence

$$
0 \rightarrow C^{*}(J) \rightarrow C^{*}(\Omega) \rightarrow C^{*}\left(G_{\Omega}\right) \rightarrow 0 .
$$

The structure of $G_{\Omega}$ is described in Proposition 3.6, and so it remains to identify $J$ and $C^{*}(J)$. (We might equally have considered the homomorphism $\chi: l^{1}(\Omega) \rightarrow l^{1}\left(G_{\Omega}\right)$ but the 
kernel of this mapping does not have a pleasant description). We omit the elementary proof of the following lemma.

Lemma 3.7. Let $\tau: S \rightarrow T$ be a semigroup homomorphism with linear extension $\tau: \mathbb{C S} \rightarrow \mathbb{C} T$. Then the kernel of $\tau$ in $\mathbb{C} S$ is the linear span of $\left\{s-s^{\prime}: s, s^{\prime} \in S, \tau(s)=\tau\left(s^{\prime}\right)\right\}$.

We denote by $\mathscr{M}$ the algebra of all complex matrices indexed by $\mathbb{Z}_{+}$which are row finite and column finite. Thus $\mathscr{M}$ is just the linear span of $\left\{E_{i j}: i, j \in \mathbb{Z}_{+}\right\}$where $E_{i j}$ is the usual elementary matrix with 1 in position $(i, j)$ and 0 elsewhere. We remark that the lemma below may be generalised to any field $F$.

Lemma 3.8. Let $\Omega=B R(T, \alpha)$ be a simple inverse $\omega$-semigroup and let $\chi: \mathbb{C} \Omega \rightarrow \mathbb{C} G_{\Omega}$. Then $\operatorname{ker} \chi$ is star isomorphic to $\mathbb{C} T \otimes \mathscr{M}$.

Proof. Let $\phi: \mathbb{C} T \otimes \mathscr{M} \rightarrow \mathbb{C} \Omega$ be the linear mapping determined by

$$
\phi\left(t \otimes E_{i j}\right)=(t, i, j)-(\alpha t, i+1, j+1) .
$$

Evidently $\phi$ has range in $\operatorname{ker} \chi$. To see that $\phi$ is one-one, suppose that

$$
\phi\left(\Sigma m_{t i j} t \otimes E_{i j}\right)=0 .
$$

Since $\alpha T \subset G_{0}$ we must have $\xi_{t i j}=0$ for $t \in T \backslash G_{0}$. Order $\mathbb{Z}_{+} \times \mathbb{Z}_{+}$lexicographically, and let $(m, n)$ be minimal amongst the $(i, j)$ with $\xi_{t i j}=0, t \in G_{0}$. We must surely have $\xi_{t m n}=0$ for any $t \in G_{0}$, and so it follows that all $\xi_{t i j}=0$, i.e. $\phi$ is one-one. To show that $\operatorname{ker} \chi$ is the range of $\phi$ it is enough to show that $s-s^{\prime} \in \operatorname{im} \phi$ whenever $s, s^{\prime} \in \Omega$ with $\chi(s)=\chi\left(s^{\prime}\right)$. By Lemma 3.4(ii) we may suppose that $s=(u, m, n), s^{\prime}=(v, m+k, n+k)$ where $\alpha^{r} v=\alpha^{k+r} u$ for some $r \in \mathbb{Z}_{+}$. (The idempotent $f$ in Lemma 3.4(ii) may here be replaced by $\alpha f_{1}$ if necessary). Then

$$
s-s^{\prime}=\sum_{i=0}^{k+r-1} \phi\left(\alpha^{i} u \otimes E_{m+i n+i}\right)-\sum_{j=0}^{r-1} \phi\left(\alpha^{j} v \otimes E_{m+k+j n+k+j}\right) .
$$

Note that

$$
\begin{aligned}
\phi\left(\left(t \otimes E_{i j}\right)^{*}\right) & =\phi\left(t^{*} \otimes E_{j i}\right) \\
& =\left(t^{*}, j, i\right)-\left(\alpha\left(t^{*}\right), j+1, i+1\right) \\
& =\left(t^{*}, j, i\right)-\left((\alpha(t))^{*}, j+1, i+1\right) \\
& =\left(\phi\left(t \otimes E_{i j}\right)\right)^{*} .
\end{aligned}
$$

To complete the proof it is enough to show that $\phi$ is multiplicative on the basic tensors $t \otimes E_{i j}$ 
The multiplication in $\mathbb{C} T \otimes \mathscr{M}$ is determined by

$$
\left(u \otimes E_{i j}\right)\left(v \otimes E_{m n}\right)=\left\{\begin{array}{cl}
u v \otimes E_{i n} & \text { if } j=m \\
0 & \text { otherwise }
\end{array} .\right.
$$

For $j=m$ we have

$$
\begin{aligned}
\{(u, i, j) & -(\alpha u, i+1, j+1)\}\{(v, m, n)-(\alpha v, m+1, n+1)\} \\
& =(u v, i, n)-(\alpha u \alpha v, i+1, n+1)-(\alpha u \alpha v, i+1, n+1)+(\alpha u \alpha v, i+1, n+1) \\
& =(u v, i, n)-(\alpha(u v), i+1, n+1) .
\end{aligned}
$$

For $j=m+r, r \in \mathbb{N}$ we have

$$
\begin{aligned}
\{(u, i, j) & -(\alpha u, i+1, j+1)\}\{(v, m, n)-(\alpha v, m+1, n+1)\} \\
& =\left(u \alpha^{r} v, i, n+r\right)-\left(u \alpha^{r}, i, n+r\right)-\left(\alpha u \alpha^{r+1} v, i+1, n+r+1\right)+\left(\alpha u \alpha^{r+1} v, i+1, n+r+1\right) \\
& =0
\end{aligned}
$$

and similarly we obtain zero product in the remaining case. The proof is complete.

Since $\mathscr{K}$ is a type I $C^{*}$-algebra there is a unique $C^{*}$-tensor product of $C^{*}(T)$ with $\mathscr{K}$. We shall denote the product by $C^{*}(T) \otimes \mathscr{K}$, since we shall never need to consider the pure algebraic tensor product of $C^{*}$-algebras.

Theorem 3.9. Let $\Omega=B R(T, \alpha)$ be a simple inverse $\omega$-semigroup. Then there is a short exact sequence

$$
0 \rightarrow C^{*}(T) \otimes \mathscr{K} \rightarrow C^{*}(\Omega) \rightarrow C^{*}\left(G_{\Omega}\right) \rightarrow 0 .
$$

Proof. All that remains to prove is that $C^{*}(\mathbb{C} T \otimes \mathscr{M})=C^{*}(T) \otimes \mathscr{K}$; this is a straightforward exercise, for example by using the fact that $\mathscr{K}$ is the direct limit of a chain of finite dimensional matrix algebras. Alternatively one may use fact (3) for $B G^{*}$ algebras, the easy fact that $\mathscr{M}$ is a $B G^{*}$-algebra and the fact that $C^{*}(A \otimes B)=$ $C^{*}(A) \otimes_{\max } C^{*}(B)$ for $B G^{*}$-algebras (see e.g. [11]).

Our discussion of the weak containment problem for $B R(T, \alpha)$ hinges on the following diagram.

$$
\begin{gathered}
0 \rightarrow C^{*}(T) \otimes \mathscr{K}^{\Phi} \rightarrow C^{*}(\Omega) \stackrel{x}{\rightarrow} C^{*}\left(G_{\Omega}\right) \rightarrow 0 \\
\downarrow \lambda_{1} \times l \quad \downarrow \lambda \quad \downarrow \lambda \quad \downarrow \lambda_{2} \\
C_{l}^{*}(T) \otimes \mathscr{K} \stackrel{\Phi}{\rightarrow} C_{l}^{*}(\Omega) \stackrel{\bar{x}}{\rightarrow} C_{l}^{*}\left(G_{\Omega}\right)
\end{gathered}
$$


In the diagram $\phi$ is the extension of the monomorphism given in Lemma 3.8, $\lambda_{1}, \lambda_{2}$ are respectively the left regular representations of $T, G_{\Omega}, l$ is the identity mapping and $\bar{\chi}$ is the extension of $\chi$ guaranteed by Proposition 1.4. We show below that the closure of $\mathbb{C} T \otimes \mathscr{M}$ in $C_{l}^{*}(\Omega)$ can be identified with $C_{l}^{*}(T) \otimes \mathscr{K}$ and hence we obtain the mapping $\phi$. The commutativity of the diagram and the exactness of the top row enables us to characterise weak containment for $\Omega$.

Theorem 3.10. Let $\Omega=B R(T, \alpha)$ with $T=\bigcup_{i=0}^{d-1} G_{i}$. Then $\Omega$ has weak containment if and only if some $G_{i}$ is amenable (equivalently all $G_{i}$ are amenable).

Proof. We employ notation as in Lemma 3.8. Let $a=\phi\left(t \otimes E_{i j}\right)$. We identify the action of $\lambda(a)$ on $l^{2}(\Omega)$. In $\Omega$ we have

$$
\begin{gathered}
(t, i, j)^{*}(t, i, j) \geqq(u, m, n)(u, m, n)^{*} \\
\Leftrightarrow\left(t^{*} t, j, j\right) \geqq\left(u u^{*}, m, m\right) .
\end{gathered}
$$

For $m=j+r, r \in \mathbb{N}$ this holds for all $u$; for $m=j$ this holds if and only if $t^{*} t \geqq u u^{*}$ in $T$. For such $(u, m, n)$ we have

$$
\lambda(t, i, j)(u, m, n)=\left(\left(\alpha^{m-j} t\right) u, i-j+m, n\right)
$$

and this gives the only non-zero action of $\lambda(t, i, j)$. Since $\alpha t \in G_{0}$ we have

$$
\lambda(\alpha t, i+1, j+1)(u, m, n)=\left\{\begin{array}{cl}
\left(\left(\alpha^{m-(j+1)} \alpha t\right) u, i-j+m, n\right) & \text { if } m=j+r, r \in \mathbb{N} \\
0 & \text { otherwise }
\end{array}\right.
$$

Therefore

$$
\lambda(a)(u, m, n)=\left\{\begin{array}{cl}
(t u, i, n) & \text { if } m=j, t^{*} t \geqq u u^{*} \\
0 & \text { otherwise }
\end{array}\right.
$$

This means that for $a \in \operatorname{ker} \chi$ the action of $\lambda(a)$ on $l^{2}(\Omega)$ is equivalent to $\lambda_{1} \otimes l\left(\phi^{-1}(a)\right)$ on $l^{2}(T) \otimes l^{2}$. This gives the mapping $\phi$ and the commutativity of the left hand square in $(\dagger)$. The right hand square is clearly commutative.

Suppose that $\Omega$ has weak containment. Then $\lambda$ is one-one and so $\lambda_{1} \otimes \imath$ is one-one, $\lambda_{1}$ is one-one. Thus $T$ has weak containment and so each $G_{i}$ is amenable (by the finite analogue of Theorem 2.7).

Suppose that some $G_{i}$ is amenable, and hence all $G_{i}$ are amenable. Then $G_{\Omega}$ is amenable by Proposition 3.6(ii) and so $\lambda_{2}$ is one-one. Also $T$ has weak containment so that $\lambda_{1}$ is one-one. By Sakai [9, Proposition 1.22.9] $\lambda_{1} \otimes \imath$ is one-one. Since the top row of $(\dagger)$ is exact it follows that $\lambda$ is one-one, i.e. $\Omega$ has weak containment.

Remark. Theorem 3.9 provides a short exact sequence for $C^{*}(\Omega)$ that highlights the canonical homomorphism $\chi: \Omega \rightarrow G_{\Omega}$. By the methods of this section one readily provides 
corresponding short exact sequences for any inverse $\omega$-semigroup, viz.

$$
0 \rightarrow I \rightarrow \mathbb{C} \Omega \rightarrow \mathbb{C} G_{\Omega} \rightarrow 0 .
$$

When $\Omega$ is as in Theorem 3.2, with $\Omega_{k}=B R(T, \alpha)$, we have

$$
I=\mathbb{C} G_{0} \oplus \mathbb{C} G_{1} \oplus \cdots \oplus \mathbb{C} G_{k-1} \oplus \mathbb{C} T \oplus \mathscr{M} .
$$

When $\Omega$ is a Clifford semigroup, $\Omega=\cup\left\{G_{n}: n \in \mathbb{Z}_{+}\right\}$we have

$$
I=\stackrel{\oplus}{ } \mathbb{C} G_{n} \text {. }
$$

The key observation in each case is that for $i \neq j$

$$
\left(g_{i}-e_{i+1} g_{i}\right)\left(g_{j}-e_{j+1} g_{j}\right)=0 .
$$

The algebraic short exact sequences extend in the obvious way to $C^{*}$-algebra short exact sequences.

\section{REFERENCES}

1. B. A. Barnes, Representations of the $l^{1}$-algebra of an inverse semigroup, Trans. Amer. Math. Soc. 218 (1976), 361-196.

2. B. A. Barnes, Representations of the $l^{1}$-algebra of an inverse semigroup having the separation property, Glasgow Math. J. 18 (1977), 131-143.

3. J. Duncan and I. Namioka, Amenability of inverse semigroups and their semigroup algebras, Proc. Roy. Soc. Edinburgh 80A (1978), 309-321.

4. J. M. Howle, An introduction to semigroup theory (Academic Press, London, 1976).

5. D. B. McAlister, A random ramble through inverse semigroups, Semigroups (edited by T. E. Hall, P. R. Hones and G. B. Preston) (Academic Press, New York, 1980), 1-25.

6. W. D. MunN, Regular $\omega$-semigroups, Glasgow Math. J. 9 (1968), 46-66.

7. T. W. PALmer, Monograph on general *-algebras; in preparation.

8. A. L. T. Paterson, Weak containment and Clifford semigroups, Proc. Roy. Soc. Edinburgh 81A (1978), 23-30.

9. S. SAKAI, C*-algebras and $\mathrm{W}^{*}$-algebras (Springer, Berlin, 1971).

10. J. R. Wordingham, The left regular *-representation of an inverse semigroup, Proc. Amer. Math. Soc. 86 (1982), 55-58.

11. J. R. Wordingham, Topics in semigroup algebras (Ph.D. thesis, University of Stirling, 1982). 